\title{
Basic Emotions, Complex Emotions, Machiavellian Emotions ${ }^{1}$
}

\author{
Paul E. Griffiths, \\ Department of History and Philosophy of Science, \\ University of Pittsburgh, \\ Pittsburgh, \\ PA 15232, \\ USA \\ pauleg@pitt.edu
}

\begin{abstract}
The current state of knowledge in psychology, cognitive neuroscience and behavioral ecology allows a fairly robust characterization of at least some, so-called 'basic emotions' - short-lived emotional responses with homologues in other vertebrates. Philosophers, however are understandably more focused on the complex emotion episodes that figure in folk-psychological narratives about mental life, episodes such as the evolving jealousy and anger of a person in an unraveling sexual relationship. One of the most pressing issues for the philosophy of emotion is the relationship between basic emotions and these complex emotion episodes. In this paper, I add to the list of existing, not necessarily incompatible, proposals concerning the relationship between basic emotions and complex emotions. I analyze the writings of 'transactional' psychologists of emotion, particularly those who see their work as a contribution to behavioral ecology, and offer a view of the basic emotion that focuses as much on their interpersonal functions as on their intrapersonal functions. Locating basic emotions and their evolutionary development in a context of processes of social interaction, I suggest, provides a way to integrate our knowledge of basic emotions into an understanding of the larger emotional episodes that have more obvious implications for philosophical disciplines such as moral psychology.
\end{abstract}

11,416 words including abstract, notes and references

\footnotetext{
${ }^{1}$ In preparing this paper I am indebted to comments on an the draft delivered to Royal Institute of Philosophy 2001 at the University of Manchester and later that summer to a seminar in the Department of Philosophy, Kings College London.
} 


\section{Emotion Episodes}

According to the distinguished philosopher Richard Wollheim, an emotion is an extended mental episode that originates when events in the world frustrate or satisfy a pre-existing desire (Wollheim, 1999). This leads the subject to form an attitude to the world which colors their future experience, leading them to attend to one aspect of things rather than another, and to view the things they attend to in one light rather than another. The idea that emotions arise from the satisfaction or frustration of desires - the 'match-mismatch' view of emotion etiology - has had several earlier incarnations in the psychology of emotion $^{2}$. Early versions of this proposal were associated with the attempt to replace the typology of emotion found in ordinary language with a simpler theory of drives and to define new emotion types in terms of general properties such as the frustration of a drive. The match-mismatch view survived the demise of that revisionist project and is found today in theories that accept a folk-psychological-style taxonomy of emotion types based on the meaning ascribed by the subject to the stimulus situation. For example, the matchmismatch view forms part of the subtle and complex model of emotion episodes developed over many years by Nico Frijda (Frijda, 1986). According to Frijda, information about the 'situational antecedents' of an emotion - the stimulus in its context, including the ongoing goals of the organism - is evaluated for its relevance to the multiple concerns of the organism. Evaluation of match-mismatch - the degree of compatibility between the situation and the subject's goals - forms part of this process.

\footnotetext{
${ }^{2}$ See, for example, (Mandler, 1984).
} 
The result of the evaluation process is an understanding of the situation in terms of the possible actions it affords and the urgency of adopting a course of action. This understanding may in turn initiate physiological changes readying the organism for action and the formation of dispositions to act on various anticipated contingencies. Each stage of the emotion process is regulated by cognitive activity outside the emotion process itself, and the whole emotion process operates in a 'continual updating' mode leading to a varied emotion episode, rather than 'running its course' to result in a single emotion. Many other 'cognitive appraisal' theories of emotion share Frijda's conception of an ongoing process of evaluation with feedback and hence are theories of emotion episodes rather than theories of the elicitation of a single emotion. But at the heart of all these models are claims about the features of the emotion-eliciting situation that lead to the production of one emotion or another at some point in the episode. These claims are usually expressed as a set of dimensions against which the situation is assessed, one of which often corresponds to match-mismatch. Many theorists label points in the resulting evaluation hyperspace with the names of emotion categories, which would seem to imply that the type-identity of an emotion is determined by the evaluation process ${ }^{3}$.

Research in the 'dimensional appraisal' tradition consists mainly in documenting the association of regions in the hyperspace defined by the proposed dimensions of evaluation with particular emotional responses. Frijda's model has been criticized for its very comprehensiveness - its desire to account for every finding documented in this rich

\footnotetext{
${ }^{3}$ For a review of appraisal theories, see (Scherer, 1999).
} 
empirical literature (Scherer, 1999). This form of criticism is well known to philosophers of science from the example of Darwin's 1868 theory of pangenesis. A comprehensive theory that fits all the known data is unable to perform one of the vital functions of theory, which is to contradict the 'facts', leading to their reexamination and the progressive transformation of the empirical base. In contrast to Darwin's theory of pangenesis, Mendelian genetics contradicted not only much accepted low-level theory about heredity but also contradicted what appeared to be the simple, factual outcome of many breeding experiments. One might hope that a psychological theory of emotion would have the same effect - leading us to reexamine some of our existing beliefs.

Appraisal theorists have also become sensitive to the charge that their models are not based on the reality of emotion processes, but rather on the image of those processes recorded in folk-wisdom. This is because appraisal models have traditionally been tested by asking people who have experienced a particular emotion to report on the appraisal process, or even by asking people to report on the relevance of certain dimensions of evaluation to certain emotion concepts. This comes close to 'conceptual analysis by numbers' or, as the leading appraisal theorist Klaus Scherer has expressed it, to studies that "do little more than explicate the implicational semantic structures of our emotion vocabulary" (Scherer, 1999: 655). This challenge to appraisal theory can be met in a number of ways, including studies that manipulate situational factors relevant to the dimensions of appraisal and predict the resultant change in emotion, and studies that rely on objective measures of emotion rather than self-report. The ongoing effort to test appraisal theories as theories of emotion, rather than as elucidations of folk theory, has 
led to a consensus that emotions do not walk in step with cognitive evaluation of the stimulus unless the notion of 'cognitive evaluation' is broadened to include sub-personal processes (Teasdale, 1999). Appraisal theorists have come to accept that even such apparently conceptually complex dimensions of evaluation as Richard Lazarus's 'core relational themes' (Lazarus, 1991) can be assessed: 1. Without the information evaluated being available to other cognitive processes, 2 . Before perceptual processing of the stimulus has been completed, and 3 . Using only simple, sensory concepts to define the property that has to be identified. Some evidence supporting such 'multi-level appraisal theories' will be considered at more length in section three, as will their philosophical implications.

\section{Basic Emotions}

The emotion episodes which are the main focus of Wollheim's work, and that of other well-known philosophers ${ }^{4}$, are very different entities from the most intensively studied emotions - the so-called 'basic emotions' of the Tomkins-Izard-Ekman tradition (Griffiths, 2001). Research on the basic emotions began in the 1860s with Darwin's efforts to reveal the 'true and original' forms of human emotional behavior. Having found painting and sculpture too dominated by convention to be of any use for this purpose, he took the innovative step of using photographs to establish which facial expressions were reliably recognized as indicating certain emotions by men and women in England.

Darwin's The Expression of the Emotions in Man and Animals (Darwin, 1872) is illustrated with many of the wonderful images he used in these experiments, some taken

\footnotetext{
${ }^{4}$ e.g. (Greenspan, 1988; Greenspan, 1995; Nussbaum, 2001)
} 
from life and others posed by hired actors. Then, as he so often did, Darwin used his network of correspondents across the world to extend his investigations. In search of indigenous peoples not corrupted by exposure to European facial expressions Darwin contacted colonists at the edges of European expansion. In Australia, for example, he contacted a missionary in 'a remote part of Gippsland' and another correspondent who had ventured 'several hundred miles in the interior of Queensland'. Neglected for decades, Darwin's ideas on emotion were revived by animal behaviorists like Konrad Lorenz in the 1950s and were spectacularly confirmed in the 1960s. In one famous series of experiments the American psychologist Paul Ekman, again searching for subjects not exposed to European cultural conventions, worked amongst the Fore people of the New Guinean highlands. Using an ingenious experimental design that avoided the problem of translating the names of emotions into another language Ekman showed his subjects photographs of actors posing facial expressions associated with certain emotions. Then he asked them to pick out the face of a character in a story - a man sitting at the bedside of his dead child, for example, or a man unexpectedly confronted by a wild pig. The Fore informants reliably identified the correct faces - those Westerners would label as sadness and fear. Ekman also filmed the faces of Fore people acting out some of the same incidents and students back in the United States proved equally adept at identifying the intended emotion from these films (Ekman, 1972). At around the same time, human ethologists demonstrated the early emergence of some of these expressions in human infants (Eibl-Eibesfeldt, 1973) and primatologists reasserted the homology between human facial expressions and those of non-human primates (Chevalier-Skolnikoff, 1973). 
So for the past thirty years, there has been a consensus that certain 'basic emotions' are found in all human cultures. These are commonly called fear, anger, disgust, sadness, joy and surprise (not to be confused with the simpler, reflex-like startle response). Naturally enough, when used in this context all these emotion words refer to phenomena less rich and varied than those they refer to in common speech. Each basic emotion has a distinctive facial expression and for most of them there is evidence of distinctive physiological responses, distinctive changes in the voice and evidence of cognitive phenomena like focusing attention on the emotion stimulus. Psychologists have disputed whether the basic emotions are really basic, that is, whether the other emotions are really all based on these six. They have also disputed whether the basic emotions are emotions, suggesting instead that they are mere building blocks that form parts of more complex psychological states, and that it is these complex states that better deserve the name 'emotions'. Emotions or not, however, the basic emotions clearly form part of what is going on in emotion episodes. The characteristic facial and other behaviors associated with the basic emotions are one criterion by which people apply emotion terms. Homologous and analogous states in animals are normally called emotions and both biologists and neuroscientists take it for granted that human emotions are some kind of elaboration of these animal emotions. Finally, the basic emotions are almost the only affective phenomena about which there is a strong consensus in the scientific literature. A philosophical theory of emotion must have some way, however dismissive, of accommodating these empirical findings. My own view is that rather than dismissing them, we can build on these findings about basic emotions to obtain insights into the nature of the more complex emotions that are of primary interest to philosophers. 


\section{3. 'Affective primacy' and 'twin-pathway' models of emotion}

A controversial claim associated with research into the basic emotions is the 'affective primacy thesis'. Affective primacy means that emotional responses are independent of the rational evaluations we make of things; that we can be afraid of things that we know are not dangerous and angry about things we firmly believe to be just. In contrast, the 'cognitive' tradition in the philosophy of emotion has treated the connections between emotion and beliefs and desires about as set of conceptual truths (Deigh, 1994; Griffiths, 1989). Robert Solomon states that:

"all emotions presuppose or have as their preconditions, certain sorts of cognitions - an awareness of danger in fear, recognition of an offense in anger, appreciation of someone or something as lovable in love. Even the most hardheaded neurological or behavioral theory must take account of the fact that no matter what the neurology or the behavior, if a person is demonstrably ignorant of a certain state of affairs or facts, he or she cannot have certain emotions." (Solomon, 1993: 11).

Many psychologists, however, claim to have demonstrated experimentally that emotions can occur in the absence of the relevant cognitions. The best known of these is Robert Zajonc, who showed that subjects can form preferences for stimuli to which they have been have been exposed subliminally so that their ability to identify those stimuli remains at chance levels (Zajonc, 1980). Many results have since been obtained which confirm Zajonc's discovery. Arne Öhman and his collaborators have conditioned subjects to dislike angry faces and subsequently elicited the conditioned emotional response when 
those angry faces were masked by neutral faces so that subjects reported no conscious experience of them (Esteves \& Öhman, 1993; Öhman, 1986). In a later study, subjects were exposed to subliminal images of snakes, spiders, flowers and mushrooms. Although the subjects showed no ability to identify which stimulus they had been exposed to, subjects with previously established snake phobia showed elevated skin conductance responses to the snake images and subjects with spider phobia showed this response to the spider images (Öhman \& Soares, 1994) ${ }^{5}$.

The original controversy aroused by Zajonc's results concerned whether emotions involve a 'cognitive evaluation of the stimulus' (Lazarus, Coyne, \& Folkman, 1984; Zajonc, 1984a, 1984b). It has become clear that this was not a helpful formulation, and that what is really at issue is whether the information processing that leads to an emotional response is separate from that which leads to paradigmatically cognitive processes such as conscious report and recall, and whether the two kinds of information processing are different in kind. The predominant view at the present time is that emotions involve states that are, in some sense, representational and which constitute, in some sense, an evaluation of the stimulus (Charland, 1997; Izard, 1992; Lazarus, 1999). These states, however, can occur at many 'levels' (in a sense to be clarified below) and an evaluation that leads to an emotion can be separate from, and can contradict, the evaluation of the same stimulus that is verbally reportable and integrated with the organisms other reportable beliefs. Under normal conditions, of course, the beliefs a subject has about an emotion stimulus match their emotional response to that stimulus,

\footnotetext{
${ }^{5}$ For a brief overview, see (Öhman, 2002).
} 
but this is not always the case, and the affective primacy thesis was basically correct in its assertion that even under normal conditions there are two (or more) processes going on (Ekman, 1980; Griffiths, 1990; Rozin, 1976; Zajonc, 1980). In Paul Ekman's work these ideas are embodied in his concept of an 'automatic appraisal mechanism' - a cognitive subsystem dedicated to determining whether a stimulus will elicit an basic emotion and able to operate independently of the cognitive systems that lead to conscious, verbally reportable appraisals of the same stimulus.

This 'twin-pathway' approach to the elicitation of emotion has been solidly confirmed in the case of fear by the neuroscientist Joseph LeDoux (LeDoux, 1996; LeDoux, 1993). LeDoux distinguishes between 'cognitive computations' which yield information about stimuli and the relations between them, and 'affective computations' which yield information about the significance of stimuli for the organism and lead to physiological and behavioral responses appropriate to that significance. In fear, and probably at least some other basic emotions, key aspects of affective computation occur in the amygdala. The emotional evaluation of a stimulus can be driven by inputs at various levels of analysis. At a very early stage of perceptual processing, minimally processed data from thalamic sensory relay structures follows the 'low road' to the amygdala. This is the ultimate 'quick and dirty' route to rapid emotional response. Meanwhile, perceptual information follows a slower 'high road' to the visual, auditory, somatosensory, gustatory and olfactory cortices, projections from which to the amygdala allow responses to stimuli in a single, sensory modality. Lesions to these pathways inhibit emotional responses to stimulus features in the corresponding modalities. Finally, the amygdala receives inputs 
from brain regions associated with full-blown, polymodal, perceptual representations of the stimulus situation and with memory, allowing the emotional response to be triggered by complex, contextual features of the stimulus. However it is triggered, it is the final response in the amygdala that is associated with fear conditioning, and conditioned fear responses to simple sensory-perceptual stimuli have been shown to be relatively hard to modify.

Twin (or multiple) pathway models of emotion have considerable implications for the theories of emotion episodes discussed in section one. They bolster existing concerns about the extent to which self-report data accurately reflect actual emotion processes. As Öhman puts it: "Thus, rather than being an important factor in the shaping of emotion, as assumed by most cognitively-oriented emotion theorists..., from the present perspective, conscious cognitive mechanisms enter late in the sequence of events, with the primary aim of finding some order in and evaluating what is going on. Therefore, self-reports may be a misleading route to the understanding of emotion..." (Öhman, 1999: 345). Findings like those of LeDoux have also increased the attraction of multi-level appraisal theories (Teasdale, 1999), which preserve the guiding insight that emotional states are directed onto states of affairs in the world without having to force emotions onto the procrustean bed of the traditional 'cognitive theory' of emotion.

Twin pathway models also have major implications for the philosophy of emotion, as I have argued elsewhere (Griffiths, 1990, 1997). What is at stake for philosophers is our ability to discover the nature of emotional processes by exploring the semantic relations between emotion terms. This approach rests on the idea that emotions are mental 
representations and that emotional cognition manipulates these representations on the basis of their representational content. Hence emotional processes can be explored via the semantic 'logic' of emotions. Solomon's strictures on any future neuroscience, quoted above, depend on just this assumption. But twin-pathway models suggest that emotional representations are separate from representations of the same objects used for purposes and perhaps also different in kind. The 'separateness' (e.g. modularity or informational encapsulation) of emotional representations means that the way in which emotional and other representations interact, if they interact at all, depends on details of cognitive architecture as well as on the content of the representations. This architecture, of course, cannot be determined by studying the logical relations between emotion words. If, in addition, emotional representations are different in kind from other representations, then further problems arise. Contemporary naturalized theories of mental representation envisage the existence of several grades of representation (Dretske, 1981, 1988; Millikan, 1984). Many of the fine-grained semantic distinctions we make in natural language may fail to get a grip on representational states with more coarse-grained semantics. Millikan has also suggested that primitive mental representations may unite the functions of beliefs and desires in a single, undifferentiated functional role. Stephen Stich has explored the possibility that 'sub-doxastic' mental representations may fail to respect the logical operations that we expect to govern full-fledged beliefs (Stich, 1983). A good, and close, analogy is that between emotional representations and states of the early stages of visual processing. The states of edge and motion detectors in the visual system, for example, are clearly 'representations' in some general sense of that term, but we do not expect to be able to characterize the representational content of these states using sentences of English 
while preserving all the semantic and inferential properties of those sentences! The representational content of an edge detector is only vaguely gestured at by the sentence "This is an edge" and this is not because of a lack of work on the 'logic of edges'. If emotional representations are, as research suggests, separate, and perhaps qualitatively distinct, from conscious, verbally reportable representations of the same stimuli, then traditional philosophical analysis of the 'logic' of fear and anger must be reconceived as akin to the 'logic' of memory or the 'logic' of perception. Such analytic projects represent the elucidation of a folk theory of the mind and are potentially as important as studies of folk-physics or ethnobiology, but they bear only an indirect and problematic relationship to the psychology of emotion. Failure to distinguish between elucidating the folk theory and studying emotion processes themselves is unlikely to lead to a good account of either.

\section{Beyond the basic emotions}

After this brief sketch of the basic emotions literature, I want to explore how we might build on the understanding of these emotions to model the complex emotions that mediate human social interaction; the emotions that are of greatest interest to philosophers, particularly aestheticians and moral psychologists. Numerous suggestions already exist as to how to do this. Some contemporary evolutionary psychologists believe that we can understand human emotion by straightforwardly extending the basic emotions approach to the rest of our emotional lives. Even a person's capacity to 'experience existential dread by considering their own death' may be an adaptation to some specific problem in human evolution (Gaulin \& McBurney, 2001: 266). Following 
this strategy, David Buss argues that the brain houses specialized circuits devoted to sexual jealousy (Buss, 2000). Like the fear circuits in the amygdala, these reacts to special inputs such as unusual scents or violations of rule about personal space and uses special-purpose computational algorithms to decide that a partner is committing adultery, often far in advance of any evidence that would provide rational grounds for that belief. The jealousy module in men causes them to behave violently to their partner as a deterrent to possible adultery, and Buss has speculated that it may even contain special rules for spouse-murder. Although better founded than, for example, Victor Johnston's suggestion that women experience negative emotions during menstruation to encourage them to get pregnant next time (Johnston, 1999: 135), Buss's claims still do not have the scientific credentials of Ekman's claims about the basic emotions or LeDoux's analysis of the fear circuits. Nevertheless, the basic emotions approach has been very successful, leading to one of the few areas of consensus in the science of emotion. It is only understandable if some psychologists believe that the correct approach is more of the same. Ekman himself has suggested a more extended list of sixteen basic emotions, amusement, anger, contempt, contentment, disgust, embarrassment, excitement, fear, guilt, pride in achievement, relief, sadness/distress, satisfaction, sensory pleasure and shame (Ekman, 1999). In contrast to most other advocates of an extended basic emotions approach, however, Ekman continues to insist that basic emotions are a distinctive class of psychological phenomena marked out by their automaticity, by unique behavioral and physiological signatures and by the existence of homologous states in other primates. His believes that empirical evidence of these features will probably be forthcoming for the states on his extended list. More doctrinaire evolutionary psychologists resist the demand 
for such evidence. Steven Gaulin and Donald McBurney argue that it is inappropriate to demand that an emotion have a distinctive facial expression, since it may be more adaptive to keep emotions secret (a view discussed at more length below). They suggest that many emotions are unique to humans, have no homologues in other primates and so cannot be studied using the comparative method. Finally, they urge that the recognition of new emotional adaptations should not be prevented by the inability of current measurement techniques to identify any distinctive physiology associated with that adaptation (Gaulin \& McBurney, 2001: 264-7). While in line with the general theoretical position adopted by many contemporary evolutionary psychologists (Cosmides \& Tooby, 2000), these arguments threaten to extend the meaning of 'basic emotion' to cover just about any phenomenon in the general domain of motivation and emotion for which a plausible evolutionary rationale can be suggested. Ekman's approach has the advantage that it identifies a range of broadly comparable and individually well-characterized psychological states. I have argued elsewhere that the methodological value of a list of basic emotions is to have a list of states of more or less the same kind, so that we can look for psychological and neurological principles about states of that kind (Griffiths, 1997).

In contrast to the evolutionary psychologists, the other currently popular attempt to build a more general theory on the foundation of the basic emotions draws a fundamental distinction between 'primary' (basic) and 'secondary' emotions. This is the revival of the early C20 James/Lange theory in the work of neuroscientist Antonio Damasio and his philosophical interpreters (Charland, 1995; Damasio, 1994; Damasio, 1999; Prinz, Forthcoming). These authors have argued that the phenomenology that accompanies 
basic emotions is the perception of bodily changes caused by the subcortical circuits that drive those responses. They argue further that these 'somatic appraisals' play important functional roles in cognition and action. More complex emotions involve subtly differentiated somatic appraisals and cognitive activity realized in the neo-cortex that accompanies some combination of basic emotions. Primary emotions are part of our evolutionary inheritance, shared by all normal humans and tied to specific types of stimuli. Secondary emotions are acquired during development, show cultural and individual variation and are sensitive to more complex and abstract features of the stimulus situation. This approach identifies each emotion with one type of somatic appraisal and focuses on the functions of emotions in the internal, cognitive economy of the organism.

In this paper, however, I want to introduce and explore a very different strategy for building on the basic emotions to illuminate complex emotional episodes. The strategy draws on recent work by 'transactional' psychologists of emotion (Fridlund, 1994; Fridlund, 1989; Parkinson, 1995). In contrast to somatic appraisal theorists, these theorists focus on the functions of emotion in interactions between organisms rather than their function in the organism's internal cognitive economy. From a transactional perspective, emotions are moves people make as they negotiate how they will be treated by others and how they will think of themselves and their situation in life. Sulking, for example, in which people sabotage what would normally be mutually rewarding interactions with a social or sexual partner and reject attempts at reconciliation after conflict, can be seen as a strategy for seeking a better global deal in that particular 
relationship. People sulk because of what sulking will achieve, as much or more than because of what has happened. Interpretations of emotional behavior as 'strategic' or goal-directed behavior are a familiar feature of the literature on the 'social construction'

of emotions (Griffiths, 1997: 137-167). The work I discuss here is significantly different, because it takes a 'strategic' or goal-directed perspective on the basic emotions and locates the origins of these features of emotion in an evolutionary account of mind. I suggest that a socially-oriented ('Machiavellian') perspective on the basic emotions can be incorporated into a theory of extended emotion episodes containing many emotional and cognitive events as parts - what Ekman has called 'emotion plots' (Ekman, 1999: 55) - in such a way as to provide biological underpinnings for ideas that have traditionally been associated with social constructionist or more generally culture-based account of emotion.

\section{Emotions as Social Transactions}

There is a fundamental evolutionary puzzle about the conventional view that basic emotions have obligate facial expressions. Why would evolution produce organisms that are obliged to continually inform friend and enemy alike about their motivation and likely future behavior? As discussed above, some evolutionary psychologists have disputed Ekman's longstanding view that each evolved emotion has a distinctive facial signature. They argue that this will only be true for those emotions that it is in the interests of the organism to reveal. The best-known advocate of this view, however, is Alan Fridlund (Fridlund, 1994). Rather than arguing that there will be some emotions with facial signatures and some without, Fridlund makes a general prediction across the 
whole range of emotions that organisms will produce displays when it is advantageous for them to do so and not at other times. Emotional behaviors, he argues, are primarily signals to other organisms and as such their production takes account of the presence of other organisms and of their relationship to the organism producing the display.

Several psychologists have conducted experiments to test this perspective on emotion, mostly seeking to find 'audience effects' - cases in which social context influences whether a particular stimulus elicits emotional behavior. For example, José Miguel Fernández-Dols and María-Angeles Ruiz-Belda have documented audience effects on the production of the so-called 'true smile' - the pan-cultural expression of happiness (Fernández-Dols \& Ruiz-Belda, 1997). In a study of Spanish soccer fans they found that a wide range of facial, vocal and other behavior occurred when the favored team scored a goal. Smiles, however, occurred almost exclusively when one fan turned to another and sought to share their enthusiasm. Fernández-Dols and Ruiz-Belda found the same pattern in medal winners at the 1992 Barcelona Olympics. Gold medalists produced many signs of emotion during the medal ceremony, but smiled almost exclusively when interacting with the audience and officials. They concluded that happiness merely facilitates smiling, making it more likely to occur when the actual precipitating factor is present. That precipitating factor is a social interaction in which one person seeks to affiliate with another. Obviously, people do smile and produce other classical emotional expressions when they are alone, but several studies suggest that they do so much less often than we suppose. Even such apparently reflexive displays as faces produced in response to tastes and smells appear to be more marked in a social setting than in solitary subjects 
(Fridlund, 1994: 155-7). There are also different ways of being 'alone'. Fridlund has shown that solitary subjects who are mentally picturing themselves as taking part in a social interaction produce more emotional facial signals than subjects thinking only of the emotional stimulus and how it makes them feel. Fridlund has described this as ' implicit sociality' and remarked that his subjects display to the 'audience in their heads'(Fridlund, 1994; Fridlund et al., 1990).

Experiments like these are open to objection that they merely reveal the operation of what Ekman has termed terms 'cultural display rules'. According to the display rule conception, the occurrence of an emotion always initiates a set of expressive movements, give or take a few caveats about stimulus intensity, but subjects sometimes prevent those movements from actually occurring by utilizing the same muscles in a voluntary movement pattern. The operation of a display rule can become as automatic as any other, habitually performed action. Fully enculturated adults can respond to social cues that require them to modulate the expression of emotions as smoothly and unconsciously as they respond to features of the traffic when driving. In a well-known experiment, Ekman and his collaborators showed American and Japanese college students neutral and stress inducing films while they were alone in a room. The repertoire of facial behaviors shown during the stress phase by the two sets of subjects was very similar. However, when an experimenter was introduced into the room and asked questions about the subject's emotions as the stress film was shown again, the facial behavior of the Japanese diverged radically from that of the Americans (Ekman, 1971, 1972). Ekman interpreted this as the operation of a cultural display rule in Japanese subjects, a rule forbidding the expression 
of negative emotions in the presence of authority figures. In support of this interpretation, he was able to document the momentary onset of negative emotional expressions prior to formation of the characteristic final facial configuration of the Japanese subjects. This phenomenon of emotion 'leakage' helps make clear the difference between the display rule and transactionalist theories of the social modulation of emotion. For Ekman, the emotion process itself is distinct from the process of strategically modulating information flow. The automatic appraisal system which triggers an affect program takes no notice of display rules. Instead, the affect program response and the display rule compete for control of output systems. Leakage is a side effect of the intrinsically conflict-based architecture of this system for controlling emotional behavior. For the transactionalist, however, the strategic modulation of information flow is an intimate part of the emotion process itself. If leakage occurs, it must have some strategic function.

There is a standard transactionalist account of the strategic function of 'leakage', an account that can be traced back to the work of the ethologist Robert A Hinde (Hinde, 1985a, 1985b). Hinde's flagship examples are threat displays in birds, which, he argued, are adaptive either as bluffs or because the display elicited in response provides information that the first bird can use to assess its options. In neither case is the probability of the threat display, or its intensity, a simple consequence of the probability that the bird will attack (or, anthropomorphically, of 'how angry it is'). Hinde used a distinction between emotional 'expression' and emotional 'negotiation' to mark the difference between his view of emotion signals and the views of earlier ethologists (Hinde, 1985a, 1985b). Emotional displays seen as expressions of emotion are 
unconditional predictors of future behavior, in the sense that they reveal a motivational state that will persist and explain the future behavior. Emotional displays seen as negotiation are conditional predictors of behavior. They predict how the first organism will behave if one or more organisms make one or other of a range of possible response. The second kind of display does not reveal an enduring motivational state, because the organism's future emotional state will depend on how other organisms respond to the display. Hinde suggested that "emotional behavior may lie along a continuum from behavior that is more or less expressive to behavior concerned primarily with a process of negotiation between individuals.' (Hinde, 1985a: 989). Fridlund compares these ethological ideas to a study on human lying which contrasted two conditions, one in which subjects lied to keep secret a surprise birthday party, and one on which they lied to avoid telling someone the painful truth. In the latter condition, but not the former, subjects equivocated (Bavelas, Black, Chovil, \& Mullett, 1990). Fridlund draws a parallel between the ambiguous speech of these human agents, who are conflicted as to whether to tell the truth or to avoid an unpleasant personal interaction, and animals unsure whether to flight or flee. Both use ambiguous signals of intention to probe the likely response of the audience to an action. From this perspective 'leakage' is not the result of the architecture of the brain, but an adaptive behavior in which animals both convey information to others and obtain information that helps them form a more definite motivation.

Since Hinde wrote, the concept of 'Machiavellian intelligence' has moved to center stage in discussions of the evolution of human cognition (Byrne \& Whiten, 1988; Whiten \& 
Byrne, 1997). Intelligence is 'Machiavellian' to the extent that the evolutionary forces which shaped it concern social competition within primate groups. Machiavellian intelligence is the result of an intra-species arms race in which increased intelligence at the level of the population merely raises the bar for success at the level of the individual. Hinde's seminal papers were a response to developments in behavioral ecology in the 1970s, particularly in the theory of animal signaling, which prefigure the Machiavellian intelligence concept. His concept of emotional negotiation and the general idea that emotions are social transactions are therefore very naturally regarded as applications of the Machiavellian intelligence perspective to emotion, an idea embodied in the title of this paper. Like more traditional conceptions of intelligence, emotions are Machiavellian in a general sense simply to the extent that they find their dominant evolutionary functions in social competition. It is, however, useful to distinguish some more specific ways in which emotion may be 'Machiavellian'. A fundamental distinction, and one that is particularly useful in reconstructing the debate between Ekman and Fridlund, is between the Machiavellian expression of emotion and the Machiavellian production of emotion. It seems to be common ground, at least amongst theorists who are prepared to interpret human emotion as a product of evolution, that the expression of emotion is Machiavellian. The contextual factors that predict whether an emotion is expressed are of the sort that are likely to have been significant in human evolution - factors such as conformity to group standards and the status of the individual in the group. The sensitivity of emotional expression to such factors is very plausibly part of our evolved social competence. This need not imply, of course, that the specific rules to which individuals conform in one culture or another can be explained in evolutionary terms. 
Evolution can be equally relevant when the task is to understand how cultures generate their patterns of difference from a shared developmental system. The generic fact that there are display rules, for example, is very likely to have an evolutionary explanation. Learning to utilize evolved facial expressions appropriately in a social setting turns out to be as critical for infant monkeys as it is for infant humans (see below).

If the Machiavellian expression of emotions is common ground amongst evolutionary theorists of emotion, the Machiavellian production of emotion is a more controversial idea. A Machiavellian perspective on the production of emotion would imply something like the following:

The Machiavellian Emotion Hypothesis: Emotional appraisal is sensitive to cues that predict the value to the emotional agent of responding to the situation with a particular emotion, as well as cues that indicate the significance of the stimulus situation to the agent independently of the agent's response.

Put in the language of appraisal theories, the hypothesis is that the appraisal hyperspace has 'strategic' dimensions. Current appraisal theories identify multiple dimensions that assess the organism-relative significance of what has happened. The Machiavellian emotions hypothesis predicts that there will also be dimensions that assess the payoff to the organism of having the emotion. Putting the hypothesis in more philosophical terms, the emotional appraisal ascribes to the environment the property of affording a certain strategy of social interaction. This process, like the process described by Frijda, might 
operate in ' continuous updating mode' leading to a continuous modulation of the organisms strategies of interaction.

A Machiavellian theory of the production of basic emotions might apply to any or all levels of appraisal. First, the triggering of basic emotions via the slower, 'high road' structures might display an evolved sensitivity to social context. Although this idea seems plausible I am going to put less emphasis on it, for two reasons. First, I suspect that the behavioral consequences of this high-level process would be very be hard to distinguish empirically from the operation of complex display rules or from the effect of broader aspects of the psychology of emotion that fall under the general rubric of 'coping processes'. Second, I will suggest below that some of the best evidence for a Machiavellian perspective on the production of emotions comes from work on nonhuman animals, work which probably illuminates the 'low road' to emotion. I will concentrate, therefore, on the idea that the 'low road'- Ekman's 'automatic appraisal mechanism' - may display an evolved sensitivity to social context that is Machiavellian in nature.

Most of the evidence so far produced by transactional psychologists working with human subjects can be accounted for by a Machiavellian perspective on the expression of emotion, without endorsing the more radical thesis of Machiavellian production.

Research on audience effects is intrinsically unsuited to distinguishing the hypothesis that an emotion does not occur in inappropriate social contexts (Machiavellian production) from the hypothesis that it is not expressed in those contexts (Machiavellian expression). 
As I have sketched above, the existence of emotion 'leakage' does not straightforwardly discriminate between these two interpretations. Nor does the persistence of reduced levels of emotional behavior in asocial settings, which can be accounted for by Fridlund's concept of 'implicit sociality' (displaying to the audience in your head), an explanation that has some empirical support. Studies of the eliciting conditions for emotions are more likely to be able to discriminate between Machiavellian expression and Machiavellian production. One intriguing study, based on retrospective self-report of actual emotion episodes, found that the occurrence of anger rather than sadness as the response to a loss was predicted, not only by traditionally recognized factors such as intentional action by a human agent or breach of a norm of behavior, but also by the possibility of obtaining restitution or compensation, a finding that seems to fit the Machiavellian emotion hypothesis (Stein, Trabasso, \& Liwag, 1993). However, the best evidence I can currently find for the existence of Machiavellian factors in emotion production comes from studies on non-human animals. These studies also suggest that Ekman's concept of a display rule needs to be amended in a way that makes even Machiavellian expression a more integral part of the actual emotion process that it at first seems.

\section{Machiavellian Emotion in Animals}

Audience effects are common in animals. In one well-known study, Peter Marler and Christopher Evans found sophisticated audience effects in Golden Sebright chickens. These birds give two alarm calls, one for aerial predators and another for terrestrial predators. Although solitary chickens are clearly afraid when they see an aerial predator, they do not produce the relevant alarm call. Likewise, male chickens call excitedly when 
they find food, but only if there are female chickens in the vicinity. The evolutionary rationale for these audience effects is obvious: there is no point warning chickens who aren't there or demonstrating foraging ability to other males. Marler and Evans say that their findings are compatible with Ekman's concept of display rules. The solitary chickens show many other signs of fear when they see an aerial predator, and they may still be excited by finding food even when they produce no calls. But the very application of the display rule concept to chickens involves a very significant revision of that concept. Display rules those were originally introduced as 'learnt, cultural display rules' (Ekman, 1972). Their function was to explain how earlier researchers had been misled about the extent of cultural variability in human emotion, as gauged by facial behavior. The suggestion was that basic emotions and their facial displays are part of humanity's evolutionary heritage, but are modified differently in every culture as a result of social learning. The chicken 'display rules', however, are not culture-specific but speciestypical, and it is most unlikely that their development in individual chickens requires learning in the sense that a human infant might be supposed to acquire a display rule by imitation or by reinforcement of initial performances. In Marler and Evans's usage, the concept of a display rule has is reduced to marking the bare distinction between Machiavellian expression and Machiavellian emotion outlined in the previous section. The chicken's appraisal of what it has found as a high value food item and its consequent emotional state have an existence independent of the chicken's Machiavellian decision to reveal this emotion to conspecifics. 
I will suggest below that the distinction between having an emotion and expressing it may be distinctly problematic in non-human subject. But even if the role of Machiavellian processes in simple minds is restricted to the expression of independently existing emotional states, this carries an important message for the study of human emotions. It provides a powerful argument against the idea that Machiavellian processes imply the sort of sophisticated cognitive abilities that naturally come to mind when we hear phrases like 'negotiation' and 'sensitivity to social context'. The existence of audience effects in animals is in tension with some basic stereotypes about emotion. Emotions are the paradigm of something that happens without regard for the consequences. Emotions are also stereotypically 'biological'. Something sounds right in Konrad Lorenz's epigram that animals are highly emotional people of limited intelligence: emotions are part of our 'animal nature'. Producing or suppressing behaviors so as to take account of social relationships, however, seems like a complex, cognitive achievement. It suggests processes that involve deliberation, perhaps even conscious deliberation. So the idea that the emotion system implements strategies of social interaction naturally suggests the idea that these aspects of emotion are learnt, and perhaps culture-specific, rather than being part of our evolutionary heritage. But this inference may well be entirely spurious. The existence of sophisticated audience effects in animals suggests that the social, manipulative aspects of emotion may be as evolutionarily ancient as any others. The appraisal process that sets off a transparently Machiavellian response like sulking may very well resemble the ancient, 'low road' to fear uncovered by LeDoux. 
Further support for this perspective comes from the vital role of emotion in primate social cognition. The role of experience in the development of emotional responses in primates is well known. A series of deprivation experiments conducted in the 1960s by Harry F. Harlow and his collaborators demonstrated the vital role of appropriate social contact in the development of the emotional phenotype in the rhesus macaque (Harlow, 1986). Moneys deprived of appropriate social interaction as infants are unable to interact effectively with peers, including sexual partners. An inability to respond to social contact with positive emotion, or with appropriate levels of negative emotion, seems to be an important mechanism producing these social deficits. It is also well known that lesions to the amygdala, the likely seat of much affective computation, produce severe social dysfunction in rhesus monkeys (Emery \& Amaral, 2000). These results, however, involve damage to the emotional phenotype that is too devastating to allow any evaluation of the Machiavellian emotion hypothesis, as opposed to the uncontroversial general claim that the emotions play an important role in social behavior. Other studies, however, have uncovered subtler deficits. William Mason reports that rhesus macaques deprived of social contact as infants produce a range of grossly normal facial behaviors which are generally interpreted as expressions of fear (grimace), friendliness (lipsmacking) and threat (threat faces) (Mason, 1985). What seems to be lacking in these animals is an ability to utilize these facial expressions to manage their relationships with other monkeys. Mason reports two dysfunctional patterns of behavior that are particularly interesting in the context of the present discussion. Normal monkeys use facial affiliation signals to form alliances to defeat dominant individuals and to maintain confidence in each other's support during that project. Socially deprived monkeys are unable to 
accomplish this. They also fail, unlike normal monkeys, to use facial expressions to redirect aggression from dominants against third parties. Mason explains these results in terms of the role of social experience in elaborating complex eliciting conditions for emotional behavior. Infant monkeys begin by producing these behaviors in response to relatively simple, context independent stimuli. Later on, 'As a result of functional elaborations, refinements, and transformations of the schemata [of elicitors for expressive behavior] present in early infancy, experience creates new sources of social order, new possibilities for the regulation and control of social life.' (Mason 1985: 147). The monkeys, in other words, learn to produce the same behaviors in response to subtler, context dependent stimuli, and by doing so are able to manage their social interactions with other animals in a rewarding manner.

One possible interpretation of these results is that socially skilled, adult monkeys experience emotions in the same way as the socially deprived monkeys, but have learnt to suppress them when they are socially inconvenient or fake them when they are socially useful. This interpretation confines Machiavellian social cognition to the management of social expression and excludes it from emotion production. Frankly, however, it is hard to attach any operational meaning to the idea that rhesus monkeys pretend to feel friendship for one another or pretend to be angry. It is easier to make sense of the inverse claim that they pretend not to have emotions. Perhaps some emotional responses are repressed by a relatively automatic version of a display rule, but it seems equally conceivable that the production of emotional responses is inhibited by the cues that might be supposed to figure in that display rule, so that, for instance, actions that might generate anger if 
performed by a subordinate or equal simply do not generate anger when performed by a dominant. Contextual inhibition of this kind is consistent with the neural connectivity of the primate amygdala (Emery \& Amaral, 2000: 167). The positive case, in which rhesus monkeys pretend to have emotions, is really quite implausible. When an animal produces a threat display but flees when challenged, as occurs in the examples Hinde used to introduce the idea of emotional negotiation, this can be described in functional terms as a 'bluff'. This, unfortunately, invites an anthropomorphic interpretation in which the organism is acting angry but not feeling angry. If that interpretation means anything there must be a distinction between genuine ('sincere’) agonistic displays and fakes. The very idea of human anger seems to presume a fixed relationship between the production of displays and the motivation of later behavior that runs counter to the idea of regarding an agonistic display as a behavior in its own right. Yet, in the case of animals, that is exactly what ethologists do.

The fact that the concept of emotional sincerity seems largely otiose in the study of animal cognition explains why the transactional perspective on emotion emerged quite rapidly after the abandonment of the classical ethological theory of drives. For Konrad Lorenz, an emotion was the subjective aspect of the performance of an instinctive behavior. The sequence of behavior leading up to this 'consummatory act' is driven by the accumulation of 'action-specific energy' in a reservoir. If the behavior is prevented by the presence of an inhibiting stimulus, this energy 'overflows' to produce 'displacement activities' (Lorenz, 1996). In the classic example, a cat confronting a rival but unwilling to attack begins to wash itself. In this context, unexpressed emotions serve to explain an 
apparently observable phenomena: "I think it is probable that displacements do serve a function as outlets, through a safety valve, of dangerous surplus impulses" (Tinbergen, 1952: 52). However, the drive-discharge model of instinctive behavior was rejected by most students of animal behavior by the early 1960s e.g. (Hinde, 1956). The idea of unexpressed emotions in animals came to seem like nothing more than unwarranted anthropomorphism. The complex relationship between emotional behavior and motivational states was conceptualized instead through a strategic understanding of the role of those behaviors in social interaction. In the seminal papers cited above, Hinde brought this perspective home to the study of emotion in humans.

\section{Machiavellian Emotions in Humans}

There is a straightforward evolutionary continuity argument for Machiavellian emotion production in humans. The automatic appraisal system in humans is homologous to the corresponding emotional appraisal system in other primates. If primates exhibit sensitivity to strategically significant features of social context it seems likely that the hominid line began its divergence from other primates already equipped with this ability. The Machiavellian intelligence perspective suggests that the ability to negotiate social relationships was the dominant factor driving the evolution of increased human cognitive ability. Given these background presuppositions, it seems highly unlikely that the emotion system would lose its sensitivity to social context during human evolution.

What would a Machiavellian theory of human emotion production look like? Numerous emotion theorists have suggested that emotions may be self-serving, occurring not when 
the situation objectively warrants the judgment embodied in the emotional appraisal, but rather when it suite the agent to interpret the situation in this light. Jean Paul Sartre famously took this view (Sartre, 1962). Emotions are a class of mental processes in which people regain psychic equilibrium by altering their perception of reality rather than altering reality itself. Affective cognition is thus like the fable of the sour grapes - unable to discharge the desire for the grapes by obtaining them, we discharge it by ascribing to the grapes the property of being undesirable. Likewise, according to Sartre, anger ascribes to a person the property of being hateful precisely because he stands between the agent and the satisfaction of her desires. That is the difference between the emotion of anger and rational coping with the conflicting needs of others. In anger, rather than give up some of our desires, we reinterpret the world to allow us to hang on to them. According to Sartre, the involvement of the body in emotion is a device for turning these psychic acts into involuntary happenings. Sartre compares a person in the grip of emotion to the florid hysterics of the Salpetrie. Emotions are psychosomatic symptoms used to make our pretences real to ourselves and to others. Emotions as Sartre describes them are intrinsically pathological - a form of bad faith in which people reject reality out of mental weakness. But the central insight of his theory is independent of this judgment: people can use emotions to view the world in a light that is psychologically more rewarding to us than other possible interpretations. Highly adaptive versions of this process are described in the literature on 'emotional intelligence', such as using an emotional reinterpretation of the situation to motivate oneself (Salovey, Bedell, Detweiler, \& Mayer, 2000). Viewed from the perspective of contemporary emotional intelligence literature, Sartre's work 
seems like an insightful account of human psychology marred by the French philosophers penchant for calling a spade a conspiracy against the soil.

Sartre concentrates on the intra-personal functions of emotion, in apparent contrast to the literature on strategic emotion in animals that focuses of inter-personal functions. But the difference between these two is smaller than it at first appears. Transactionalist psychologists have stressed the importance of managing self-image as well as social image in a functional way, and it is easy to see that these goals can often be accomplished simultaneously. By interpreting another's behavior as unreasonable I can both maintain my positive self-image and make an advantageous move in the social negotiation of the eventual outcome of my interaction with that person. A person who becomes angry when their sexual partner points out that they have failed to do enough around the house, for example, might gain both these advantages by focusing on the hurtful way the remark was made.

An interesting example with which to develop the Machiavellian perspective is romantic love. Most accounts of this emotion regard it as a device to create and maintain long-term pair bonds. According to Robert Frank, love is a 'commitment mechanism' a guarantee that a person will remain committed to a relationship even when temporarily more rewarding relationships become available (Frank, 1988). A special emotion is needed for this purpose, because simple means-end rationality will dictate choosing the current best option at each moment. Melvyn Konner, in contrast, has pointed out that in traditional societies few people have the option of forming a long-term pair bond on the basis of 
romantic attraction. Instead, he suggests that the irruptive, passionate love that western societies treat as the occasion for the formation of life-long partnerships may have as its primary evolutionary function motivating behaviors such as mate desertion and copulation outside the pair bond (Konner, 1982: 315-316). This suggestion has become more credible since it was first made in the light of the increasing emphasis in behavioral ecology on female promiscuity. Females in a wide range of species search for the 'best genes' independent of the need in many of the same species to maintain a stable bond with a single male to provide economic support for offspring. In humans, mate desertion and promiscuity are risky behaviors as far as immediate survival goes. They carry a high probability of agonistic interactions with other members of the group. If the advantages of these behaviors for reproductive fitness are great enough, however, love might evolve as a special motivational system designed, not to enforce commitment when impulse argues against it, but to motivate adultery when prudence argues against it. Both theories represent evolutionary just-so-stories, with all their attendant uncertainty. But if the adultery theory were correct it would dovetail interestingly with some ideas about love from the social constructionist tradition. Constructionists have emphasized the role of emotions as 'excuses' - ways to move socially sanctioned behavior into the realm of passive, involuntary, and thus excusable behavior. The existence of recognized 'excuses' also allows society to tolerate a certain amount of deviance without the complete breakdown of social norms. The use of love to excuse mate desertion is one instance to which this model could be applied. The obvious problem with the constructionist theory is that it requires both the individual emotional agent and their society to be sincerely convinced that the behavior is involuntary. In earlier work I suggested that the 
internalization of a cultural model of behavior in childhood might do the requisite work of 'naturalizing' the behavior and making its function invisible to those who enact it ${ }^{6}$. If the adultery theory of the evolution of love is correct, however, then a process of this kind would have ample biological material to work with. It would simply be a matter of hyper-cognizing a certain kind of emotional experience and establishing cultural narratives in which it figures and which would be cited to explain, and implicitly excuse, desertion. The same narratives might serve the intra-personal function of allowing the individual to regard themselves as swept along by impersonal forces and thus maintain a positive self-image in the face of the damage caused to other people by their behavior.

The ideas of the last paragraph are grossly speculative. I include them merely to make a general, theoretical point about the impact of the Machiavellian emotion perspective on emotion theory. A Machiavellian perspective on the basic emotions would allow a much tighter integration of biological and cultural theories of emotion. This would be true even if Machiavellian processes are restricted to the management of emotional expression, so long as those processes occur as an intimate part of the evolved emotion system. The basic emotions represent some of the key building blocks of complex emotion episodes. These episodes are more than just the sum of their constituent parts, but they also, inevitably, reflect the nature of those parts. What we might learn from ethological work on the Machiavellian nature of emotional behavior in animal on the one hand and social transactional account of human psychologists on the other, is to stop contrasting

\footnotetext{
${ }^{6}$ For an analysis of social constructionism about emotion, see (Griffiths, 1997, Ch 6). The idea of love as a socially accepted excuse for adultery was first suggested to me by Peter Forrest.
} 
spontaneous emotions with strategic, perhaps even manipulative, social interaction. Basic emotion processes and complex, culturally situated emotion episodes may, as it were, speak the same language. Emotion may be Machiavellian all the way down.

\section{Conclusion}

I have suggested that the basic emotions may be 'Machiavellian' in their expression and possibly also in their production, meaning that they show an evolved sensitivity to strategically significant aspects of the organism's social context. The best evidence for this, I suggest, is the presence of sophisticated social cognition in animals, where it is problematic to postulate complex psychological processes such as self-deceit and pretence. What is plausible for animal emotion, I have argued, is also plausible for lowlevel processes in human emotion. Finally, I have suggested that some of the narratives about self-serving or manipulative emotion associated with social constructionist accounts of emotion are easier to believe if these emotion episodes have biological underpinnings that take account of the organism's strategic situation at a sub-personal level. My suggestion is analogous to Alfred Mele's recent suggestion that self-deception can be generated by a set of simple cognitive biases that produce the appearance of a person choosing to believe something they know to be false (Mele, 2001). Similarly, a strategically sensitive emotion system might give rise to emotion episodes that appear self-serving and manipulative without the agent forming a plan to pursue their social interests or engage in manipulation. 


\section{Bibliography}

Bavelas, J. B., Black, A., Chovil, N., \& Mullett, J. (1990). Truth, lies and equivocations: The effects of conflicting goals on discourse. Journal of Language and Social Psychology, 9, 135-161.

Buss, D. M. (2000). The Dangerous Passion: Why Jealousy is as Essential as Love and Sex. New York: Simon and Schuster.

Byrne, R. W., \& Whiten, A. E. (1988). Machiavellian Intelligence. Oxford, New York: Oxford University Press.

Charland, L. C. (1995). Emotion as a natural kind: towards a computational foundation for emotion theory. Philosophical Psychology, 8(1), 59-84.

Charland, L. C. (1997). Reconciling Cognitive and Perceptual Theories of Emotion: A Representational Proposal. Philosophy of Science, 64(4), 555-579.

Chevalier-Skolnikoff, S. (1973). Facial expression of emotion in non-human primates. In P. Ekman (Ed.), Darwin and Facial Expression: A Century of Research in Review (pp. 11-89). New York and London: Academic Press.

Cosmides, L., \& Tooby, J. (2000). Evolutionary Psychology and the Emotions. In M. Lewis \& J. M. Haviland-Jones (Eds.), Handbook of the Emotions (2 ed., pp. 91115). New York and London: Guildford Press.

Damasio, A. R. (1994). Descartes Error: Emotion, Reason and the Human Brain. New York: Grosset/Putnam.

Damasio, A. R. (1999). The Feeling of What Happens: Body and Emotion in the Making of Consciousness. New York: Harcourt Brace. 
Darwin, C. (1872). The Expressions of Emotions in Man \& Animals ( 1st ed.). New York: Philosophical Library.

Deigh, J. (1994). Cognitivism in the theory of emotions. Ethics, 104, 824-854.

Dretske, F. (1981). Knowledge and the Flow of Information. Oxford: Blackwells.

Dretske, F. (1988). Explaining Behaviour. Cambridge, MA: Bradford/MIT.

Eibl-Eibesfeldt, I. (1973). Expressive behaviour of the deaf \& blind born. In M. von

Cranach \& I. Vine (Eds.), Social Communication \& Movement (pp. 163-194). London \& New York: Academic Press.

Ekman, P. (1971). Universals and Cultural Differences in Facial Expressions of Emotion. In J. K. Cole (Ed.), Nebraska Symposium on Motivation 4. (pp. 207-283). Lincoln, Nebraska: University of Nebraska Press.

Ekman, P. (1972). Emotions in the Human Face. New York: Pergamon Press.

Ekman, P. (1980). Biological \& cultural contributions to body \& facial movement in the expression of emotions. In A. O. Rorty (Ed.), Explaining Emotions (pp. xxx-xxx). Berkeley: University of California Press.

Ekman, P. (1999). Basic Emotions. In T. Dalgleish \& M. Power (Eds.), Handbook of Cognition and Emotion (pp. 45-60). Chichester: John Wiley and Sons Co.

Emery, N. J., \& Amaral, D. G. (2000). The role of the amygdala in primate social cognition. In R. Lane \& L. Nadel (Eds.), The Cognitive Neuroscience of Emotion (pp. 156-191). New York: Oxford University Press.

Esteves, F., \& Öhman, A. (1993). Masking the face: recognition of emotional facial expression as a function of the parameters of backward masking. Scandinavian Journal of Psychology, 34(1-18). 
Fernández-Dols, J. M., \& Ruiz-Belda, M.-A. (1997). Spontaneous facial behavior during intense emotional episodes: Artistic truth and optical truth. In J. A. Russell \& J. M. Fernández-Dols (Eds.), The Psychology of Facial Expression (pp. 255-294). Cambridge: Cambridge University Press.

Frank, R. H. (1988). Passions Within Reason: The Strategic Role of the Emotions. New York: Norton.

Fridlund, A. (1994). Human Facial Expression: An Evolutionary View. San Diego: Academic Press.

Fridlund, A. J. (1989). Evolution and facial action in reflex, social motive, and paralanguage.: University of California.

Fridlund, A. J., Schaut, J. A., Sabini, J. P., Shenker, J. I., Hedlund, L. E., \& Knauer, M. J. (1990). Audience effects on solitary faces during imagery: displaying to the people in your head. Journal of Nonverbal Behaviour, 14(2), 113-137.

Frijda, N. H. (1986). The Emotions. Cambridge: Cambridge University Press.

Gaulin, S. J. C., \& McBurney, D. H. (2001). Psychology: An Evolutionary Approach. Upper Saddle River, NJ: Prentice Hall.

Greenspan, P. (1988). Emotions and Reasons:An Inquiry into Emotional Justification. New York: Routledge.

Greenspan, P. S. (1995). Practical Guilt: Moral Dilemmas, Emotions, and Social Norms. New York: Oxford University Press.

Griffiths, P. E. (1989). The Degeneration of the Cognitive Theory of Emotion. Philosophical Psychology, 2 (3), 297-313. 
Griffiths, P. E. (1990). Modularity \& the Psychoevolutionary Theory of Emotion. . Biology \& Philosophy, 5, 175-196.

Griffiths, P. E. (1997). What Emotions Really Are: The Problem of Psychological Categories. Chicago: University of Chicago Press.

Griffiths, P. E. (2001). Emotion and Expression, International Encyclopedia of the Social and Behavioral Sciences.: Pergamon/Elsevier Science.

Harlow, C. M. (Ed.). (1986). From Learning to Love: The Selected Papers of H.F Harlow. New York: Praeger.

Hinde, R. A. (1956). Ethological Models and the Concept of 'Drive'. British Journal for the Philosophy of Science, 6, 321-331.

Hinde, R. A. (1985a). Expression and Negotiation. In G. Zivin (Ed.), The Development of Expressive Behavior (pp. 103-116). New York: Academic Press.

Hinde, R. A. (1985b). Was 'The Expression of Emotions' a misleading phrase? Animal Behaviour(33), 985-992.

Izard, C. E. (1992). Basic emotions, relations amongst emotions and emotion-cognition relations. Psychological Review, 99 (3), 561-565.

Johnston, V. S. (1999). Why we Feel: The new science of human emotions.

Konner, M. (1982). The Tangled Wing: Biological Constraints on the Human Spirit. London: William Heinemann Ltd.

Lazarus, R. S. (1991). Emotion and Adaptation. New York: Oxford University Press.

Lazarus, R. S. (1999). The cognition-emotion debate: a bit of history. In T. Dalgleish \& M. J. Power (Eds.), Handbook of Emotion and Cognition (pp. 3-19). Chichester, New York: John Wiley and sons. 
Lazarus, R. S., Coyne, J. C., \& Folkman, S. (1984). Cognition, emotion \& motivation: doctoring Humpty Dumpty. In K. Scherer \& P. Ekman (Eds.), Approaches to Emotions (pp. 221-237). Hillsdale, New Jersey: Erlbaum.

LeDoux, J. (1996). The Emotional Brain: The Mysterious Underpinnings of Emotional Life. New York: Simon and Schuster.

LeDoux, J. E. (1993). Emotional networks in the brain. In M. Lewis \& J. M. Haviland (Eds.), Handbook of Emotions (pp. 109-118). New York: Guildford Press.

Lorenz, K. (1996). The Natural Science of the Human Species: An Introduction to Comparative Behavioral Research. The Russian Manuscript (1944-1948) (R. D. Martin, Trans.). Cambridge, Mass: MIT Press.

Mandler, G. (1984). Mind and Body: The Psychology of Emotion and Stress. New York: Norton.

Mason, W. A. (1985). Experiential influences on the development of expressive behaviors in Rhesus monkeys. In G. Zivin (Ed.), The Development of Expressive Behavior (pp. 117-152). New York: Academic Press.

Mele, A.R. (2001). Self-Deception Unmasked. Princeton: Princeton University Press.

Millikan, R. G. (1984). Language, Thought \& Other Biological Categories. Cambridge, Mass.: M.I.T. Press.

Nussbaum, M. C. (2001). Upheavals of Thought : The Intelligence of Emotions. Cambridge, New York: Cambridge University Press.

Öhman, A. (1986). Face the beast and fear the face: animal and social fears as prototypes for evolutionary analyses of emotion. Psychophysiology, 23, 123-145. 
Öhman, A. (1999). Distinguishing Unconscious from Conscious Emotional Processes: Methodological Considerations and Theoretical Implications. In T. Dalgleish \& M. J. Power (Eds.), Handbook of Emotion and Cognition (pp. 321-352). Chichester: John Wiley and sons.

Öhman, A. (2002). Automaticity and the amygdala: Nonconscious responses to emotional faces. Current Directions in Psychological Science, 11(2), 62-66.

Öhman, A., \& Soares, J. J. F. (1994). Unconscious anxiety: phobic responses to masked stimuli. Journal of Abnormal Psychology, 102, 121-132.

Parkinson, B. (1995). Ideas and Realities of Emotion. London and New York: Routledge. Prinz, J. (Forthcoming). Emotional Perception. Oxford: Oxford University Press.

Rozin, P. (1976). The evolution of intelligence \& access to the cognitive unconscious. In J. M. Sprague \& A. N. Epstein (Eds.), Progress in Psychobiology \& Physiological Psychology (Vol. 6, pp. 245-281). New York: Academic Press.

Salovey, P., Bedell, B. T., Detweiler, J. B., \& Mayer, J. D. (2000). Current Directions in Emotional Intelligence Research. In M. Lewis \& J. M. Haviland-Jones (Eds.), Handbook of Emotions (2 ed., pp. 5-4-520). New York: Guildford Press.

Sartre, J. P. (1962). Sketch for a Theory of the Emotions (P. Mairet, Trans.). London: Methuen.

Scherer, K. R. (1999). Appraisal Theory. In T. Dalgleish \& M. J. Power (Eds.), Handbook of Emotion and Cognition (pp. 637-663). Chichester: New York.

Solomon, R. C. (1993). The philosophy of emotions. In M. Lewis \& J. M. Haviland (Eds.), Handbook of Emotions (pp. 3-15). New York: Guildford. 
Stein, N. L., Trabasso, T., \& Liwag, M. (1993). The representation and organization of emotional exerience: unfolding the emotion episode. In M. Lewis \& J. M. Haviland (Eds.), Handbook of Emotions (pp. 279-300). New York: Guildford Press.

Stich, S. (1983). From Folk Psychology to Cognitive Science. Cambridge, U.K.: M.I.T. Press.

Teasdale, J. D. (1999). Multi-level Theories of Cognition-Emotion Relations. In T. Dalgleish \& M. J. Power (Eds.), Handbook of Cognition and Emotion (pp. 665681). Chichester: John Wiley and sons.

Tinbergen, N. (1952). Derived activities: their causation, biological significance, origin and emancipation during evolution. Quarterly Review of Biology, 27 (1), 1-32.

Whiten, A., \& Byrne, R. W. (Eds.). (1997). Machiavellian Intelligence II: Extensions and Evaluations. Cambridge: Cambridge University Press.

Wollheim, R. (1999). On the Emotions.

Zajonc, R. B. (1980). Feeling \& thinking: preferences need no inference. American Psychologist, 35, 151-175.

Zajonc, R. B. (1984a). On the primacy of affect. In K. Scherer \& P. Ekman (Eds.), Approaches to Emotion (pp. 259-270). Hillsdale, N.J: Lawrence Erlbaum Assoc.

Zajonc, R. B. (1984b). The interaction of affect and cognition. In K. Scherer \& P. Ekman (Eds.), Approaches to Emotions (pp. 239-246). Hillsdale, NJ: Lawrence Erlbaum Associates. 